\title{
The role of TDP-43 in amyotrophic lateral sclerosis and frontotemporal dementia
}

\author{
Ian R.A. Mackenzie, MD and \\ Department of Pathology, Vancouver General Hospital, 855 West 12 $12^{\text {th }}$ Avenue, Vancouver, British \\ Columbia, V5Z 1 M9 Canada, ian.mackenzie@vch.ca, Tel: 1-604-875-4480, Fax: 1-604-875-5707 \\ Rosa Rademakers, PhD \\ Department of Neuroscience, Mayo Clinic College of Medicine, 4500 San Pablo Road, Jacksonville, \\ FL, 32224 USA
}

\begin{abstract}
Purpose of review-We examine current evidence that the TAR DNA binding protein, TDP-43, plays a pathogenic role in both amyotrophic lateral sclerosis (ALS) and frontotemporal dementia (FTD).
\end{abstract}

Recent findings-TDP-43 was recently identified as the major pathological protein in sporadic ALS and in the most common pathological subtype of FTD, frontotemporal lobar degeneration with ubiquitinated inclusions (FLTD-U). In these conditions, abnormal C-terminal fragments of TDP-43 are ubiquitinated, hyperphosphorylated and accumulate as cellular inclusions in neurons and glia. Cells with inclusions show absence of the normal nuclear TDP-43 localization. Recently, missense mutations in the gene encoding TDP-43 have been identified in patients with sporadic and familial ALS.

Summary-The recent discovery of pathological TDP-43 in both ALS and FTLD-U confirms that these are closely related conditions within a new biochemical class of neurodegenerative disease, the TDP-43 proteinopathies.

\section{Keywords}

TDP-43; amyotrophic lateral sclerosis; frontotemporal dementia; FTLD-U

\section{Introduction}

There is growing evidence that amyotrophic lateral sclerosis (ALS) and frontotemporal dementia (FTD) are closely related conditions with overlapping clinical, genetic and neuropathological features [1]. The recent identification of the transactive response (TAR) DNA binding protein with $\mathrm{M}_{\mathrm{r}} 43 \mathrm{kDa}$ (TDP-43) as the major pathological protein, in both ALS and the most common pathological subtype of FTD (frontotemporal lobar degeneration with ubiquitinated inclusions, FTLD-U) $[2 \cdot \bullet, 3 \bullet \bullet$, provides the strongest evidence to date that these conditions are part of a clinicopathological spectrum of disease. Furthermore, this discovery

Correspondence to: Ian R.A. Mackenzie.

Publisher's Disclaimer: This is a PDF file of an unedited manuscript that has been accepted for publication. As a service to our customers we are providing this early version of the manuscript. The manuscript will undergo copyediting, typesetting, and review of the resulting proof before it is published in its final citable form. Please note that during the production process errors may be discovered which could affect the content, and all legal disclaimers that apply to the journal pertain. 
provides important new insights into the pathogenesis of these conditions and the potential for the development of new diagnostic tests and therapies.

\section{TDP-43 is the pathological protein in ALS and FTLD-U}

One of the most characteristic neuropathological features of ALS is the presence of ubiquitinimmunoreactive (ub-ir) neuronal cytoplasmic inclusions (NCI) in the degenerating motor neurons [4]. A significant proportion of ALS patients develop cognitive deficits, often with prominent frontal lobe features [5], and are found to have additional ub-ir NCI and neurites in the frontotemporal neocortex and hippocampus [6,7]. Similar ub-ir, tau- and $\alpha$-synucleinnegative cortical pathology (FTLD-U) is now recognized to also be the most common pathological substrate for clinical FTD, in the absence of motor features $[8,9]$. Until recently, it was uncertain whether FTLD-U represented a single disease process, or if it included a number of discrete entities in which the ubiquitinated protein was different. The recognition of several identifiable subtypes of FTLD-U pathology, each with relatively specific clinical and molecular genetic correlations, was initially interpreted as suggesting that FTLD-U was a heterogeneous collection of diseases [10,11]. However, this issue was finally resolved, in late 2006, when two groups independently identified the pathological protein in both FTLD-U and ALS as being TDP- $43[2 \bullet \bullet, 3 \bullet \bullet]$.

In those initial studies, immunoblot analysis performed on the high $\mathrm{M}_{\mathrm{r}}$ insoluble protein fraction from postmortem FTLD-U brain tissue identified disease specific bands that were excised and analyzed by liquid chromatography-mass spectrometry $[2 \bullet \cdot 3 \bullet \bullet]$. The resulting peptides were found to correspond to amino acid sequences in the C-terminal region of TDP- 43 . Commercially available antibodies against TDP-43 were found to consistently label the ub-ir inclusions in cases of FTLD-U and also ALS (Figure 1), but not the characteristic inclusions in a variety of other neurodegenerative conditions. An interesting observation, with possible functional implications, was the fact that neurons that contained TDP-43 inclusions showed an absence of the normal diffuse nuclear staining pattern [3・•]. In addition to the normal 43 kDa band, TPD-43 immunoblot analysis of FTLD-U and ALS tissue, demonstrated disease specific bands at $\sim 25$ and $45 \mathrm{kDa}$, as well as a high $\mathrm{M}_{\mathrm{r}}$ smear (Figure 2). The pathological proteins were shown to represent abnormal C-terminal fragments of TDP-43 that were ubiquitinated and phosphorylated.

A number of subsequent studies have confirmed that most clinical and pathological subtypes of FTLD-U and ALS are characterized by TDP-43 immunoreactive inclusions $[12 \bullet \bullet, 13 \bullet, 14$, $15 \bullet \cdot, 16,17]$. Moreover, by virtue of its greater sensitivity and specificity, TDP-43 immunohistochemistry has proved to be a powerful new tool for investigating the neuropathology of these conditions, demonstrating changes that were not previously recognized. These include TDP-43-immunoreactive cytoplasmic inclusions in glial cells of presumed oligodendroglial lineage $[2 \bullet, 15 \bullet, 16,18 \bullet]$, granular neuronal cytoplasmic "preinclusions" [19], dystrophic neurites in the CA1 region in cases with hippocampal sclerosis [20] and more extensive extramotor pathology in cases of ALS, with and without dementia [19]. Antibodies raised against phosphorylated epitopes of TDP-43 have proven to be even more sensitive and specific, as they only identify the pathological forms of the protein [21•] while the use of C-terminal and $\mathrm{N}$-terminal specific antibodies has suggested that the pathological inclusions in different populations of neurons may be composed of different forms of TDP-43 [22•]. As a result, FTLD-U and ALS are now recognized as representing a clinicopathological spectrum within a new biochemical class of neurodegenerative disease, the TDP-43 proteinopathies. 


\section{Normal function of TDP-43 in nervous system}

TDP-43 is a 414 amino acid nuclear protein that is encoded by the TARDBP gene on human chromosome 1p36.2. It is highly conserved and ubiquitously expressed in a variety of tissues including brain [23]. TDP-43 contains 2 RNA-recognition motifs (RRM1: aa 106-175 and RRM2: aa 191-262) and a glycine-rich C-terminal region ( aa 274-413) that allow it to bind single stranded DNA, RNA and proteins [23,24]. It was initially cloned as a human protein capable of binding to the TAR DNA of human HIV-1, where it acts as a transcription repressor [25]. It was subsequently identified as part of a complex involved in splicing the cystic fibrosis transmembrane conductance regulator gene [23] and also the apoA-II gene [26]. The exon skipping and splicing inhibitory activity requires the glycine-rich C-terminal domain that binds to several members of the heterogeneous nuclear ribonucleoprotein (hnRNP) family [24,27]. TDP-43 has also been shown to act as a scaffold for nuclear bodies through an interaction with survival motor neuron protein [28]. It may also be involved in mRNA stability, microRNA biogenesis, apoptosis and cell division [29•]. In the brain, TDP-43 is normally localized to the nucleus of neurons and some glial cells [3••]. Although, its physiological function in the nervous system is not currently known, one recent study has suggested it may act as a neuronal activity-response factor, involved in the regulation of neuronal plasticity [30].

\section{The spectrum TDP-43 proteinopathies}

Although the initial reports suggested that pathological TDP-43 is both a specific and sensitive marker of all subtypes of FTLD-U and ALS $[2 \bullet \bullet, 3 \bullet \bullet, 13 \bullet]$, subsequent studies have identified some important exceptions. While the vast majority of sporadic FTLD-U cases are found to be TDP-43-positive, most large series have identified a small proportion in which the ub-ir pathology is negative $[12 \bullet, 20]$. Two recent papers have provided detailed description of these "atypical" cases (aFTLD-U), which represented $10-20 \%$ of all FTLD-U in the respective series $[31 \bullet, 32]$. In contrast to TDP-43-positive cases, all aFTLD-U cases were sporadic with very early onset FTD, characterized by severe progressive psychobehavioural abnormalities in the absence of significant aphasia, cognitive-intellectual dysfunction or motor features. The neuropathology consisted of NCI and unique neuronal intranuclear inclusions that were only reactive for ubiquitin. Based on the unusual and highly consistent clinical phenotype and neuropathology, the authors suggested that aFTLD-U represents a newly recognized and specific disease entity.

In familial FTLD-U, different patterns of TDP-43 pathology have been found to correlate with most of the known genetic causes, including mutations in the genes encoding progranulin and valosin-containing protein and in families with FTD and MND linked to chromosome 9p21-13 $[12 \bullet, 14,17]$. An exception is FTD linked to chromosome 3 , caused by a mutation in the gene encoding the charged multi-vesicular body protein gene $(C H M P 2 B)$, which is characterized by ub-ir NCI in hippocampal granule cells, that are not reactive for TDP-43 [12••,33•].

In ALS, TDP-43 positive inclusions, in both lower motor neurons and glia, are a consistent feature of all sporadic cases and familial cases without SOD1 mutations $[15 \bullet \cdot 16]$. However, the absence of immunohistochemical or biochemical evidence of pathological TDP-43 in human cases and animal models with SOD1 mutations suggests that neurodegeneration in these cases may have a different pathogenesis $[15 \bullet \bullet, 16,34 \bullet]$.

A number of recent studies have also raised questions about the disease specificity of TDP-43 pathology by demonstrating some degree of positivity in a variety of conditions, outside the usual spectrum of FTD and ALS. TDP-43 immunoreactivity is reported to be a consistent feature of ALS-parkinsonism-dementia complex of Guam $\left[35^{\bullet}, 36^{\bullet}\right]$ and present in a significant proportion of cases of hippocampal sclerosis dementia $[12 \bullet \bullet, 37 \bullet \bullet, 38]$, classical Pick's disease [39], corticobasal degeneration [40], Alzheimer's disease [37••,40,41], Parkinson's disease 
and dementia with Lewy bodies [41,42]. In most of these conditions, the TDP-43 pathology is anatomically restricted to mesial temporal structures, shows only partial co-localization with the other pathological changes and is of uncertain clinical relevance [37••,38-42].

It is anticipated that our understanding of the spectrum of TDP-43 proteinopathies will continue to evolve as more cases and additional conditions are examined. Rather than diminish the importance of this protein in the pathogenesis of ALS and FTD, this new information will help to clarify the relationship between different ALS and FTD subtypes and their relationship with other neurodegenerative conditions.

\section{Mutations in TARDBP cause ALS but not dementia}

Rare pathogenic missense mutations and multiplications have been identified in genes encoding the major constituents of the pathological deposits in several neurodegenerative diseases [43-45]. The gene encoding TDP-43 (TARDBP) therefore represents an excellent candidate for causing or increasing the risk to develop a disease in the spectrum of TDP-43 proteinopathies. Initial sequence analyses of TARDBP in patients with sporadic FTD and ALS, as well as familial FTD, failed to identify mutations [46]. Similarly, no evidence of genetic variation in TARDBP increasing risk for FTD or ALS was observed [46-48]. However, subsequent large population-based TARDBP mutation screenings, by us and by others, have identified 16 different missense mutations in 19 genealogically unrelated ALS patients, which were absent in healthy controls $[49 \bullet, 50 \bullet, 51 \bullet \bullet, 52,53 \bullet]$ (Figure 3). Eight mutations have been found in familial ALS patients $[49 \bullet, 50 \bullet, 51 \bullet \bullet, 52,53 \bullet]$, while eight others were identified in patients with sporadic ALS [50•,51••]. Mutations c.1077G>A (p.A315T), c.1009A>G (p.M337V) and c.1278G>A (p.A382T) were identified in two families each and haplotype sharing studies supported a common genetic origin for mutations p.M337V and p.A382T. TARDBP mutations were not restricted to Caucasians ALS populations; mutation c.892G $>\mathrm{A}$ (p.G298S) was identified in a Chinese family [53•] and c.1028A>G (p.Q343R) in a Japanese family [52].

Depending on the study, the overall TARDBP mutation frequency in ALS has ranged from 3\% to $6 \%$, with the exception of the initial report by Sreedharan et al. [51••], in which a much lower TARDBP mutation frequency of $0.6 \%$ (3/526) was identified. Both population-based studies in which sporadic mutation carriers were identified, described comparable TARDBP frequencies for sporadic and familial ALS [50•,51••]; however mutation analyses in a large clinical series of sporadic ALS patients of European descent did not identify mutations [54] and other studies have identified TARDBP mutations exclusively in familial ALS [49•,52, 53•]. Without supportive functional data, the pathogenic character of each of the TARDBP mutations identified in a single sporadic ALS patient remains uncertain.

The clinical phenotype of TARDBP mutation carriers resembles classical adult onset ALS. Based on the current published literature, the mean onset age for mutation carriers was $56.3 \pm$ 12.1 years $(\mathrm{N}=30$; range $30-83$ years) with similar onset ages in familial $(\mathrm{N}=22 ; 55.8 \pm 12.1$ years) and sporadic $(\mathrm{N}=8 ; 57.5 \pm 12.8$ years $)$ cases $[49 \bullet, 50 \bullet, 51 \bullet \bullet, 52,53 \bullet]$. The mean duration of disease was $4.3 \pm 3.6$ years ( $\mathrm{N}=18$; range $1-12$ years). Even within a single family, the age of onset varied significantly (by as much as 35 years) [53•], suggesting that additional genetic and/or environmental factors determine the disease expression of TARDBP mutations. Thirtythree percent of mutation carriers (11/33) had bulbar onset and 67\% (22/33) limb-onset ALS, a distribution comparable to non-TARDBP mutation carriers [55,56]. Interestingly, despite the common co-occurrence of cognitive symptoms in ALS patients [5], none of the TARDBP mutation carriers identified to date, have had a personal or family history of dementia. In agreement with this finding, TARDBP mutations have not been identified in patients with FTD, FTD-ALS or AD, characterized by TDP-43 pathology $[50 \bullet, 53 \bullet]$. 
Thus far, pathological examination has only been performed in two families with TARDBP mutations $[52,53 \cdot]$. The neuropathological changes in patients from both families were characteristic of ALS, with motor neuron loss, gliosis and the presence of Bunina bodies. As expected, TDP-43-positive NCIs and glial cytoplasmic inclusions were observed in the anterior horn cells of the spinal cord and in various other regions of the central nervous system. In one family the authors suggested a higher frequency of TDP-43-positive "pre-inclusions" compared to other patients with sporadic ALS [53•]. Biochemical analyses of TDP-43 protein extracted from the spinal cord of the autopsied case carrying the p.Q343R mutation showed elevated levels of the abnormal molecular-weight fragments of $\sim 25$ and $45 \mathrm{kDa}$, that were previously observed in sporadic ALS and in $S O D-1$ negative familial ALS, suggesting TARDBP mutations may accelerate the production of these fragments [52]. Whether these neuropathological findings are characteristic of all TARDBP mutation carriers needs further confirmation.

All but one of the reported mutations cluster in TARDBP exon 6, encoding the highly conserved C-terminal region of TDP-43 (Figure 3). Four mutations involve glycine residues, in close proximity of each other, in the glycine-rich region, while the other mutations affect conserved residues in the remaining portion of the $\mathrm{C}$-terminal domain. No clustering of familial versus sporadic mutations in specific regions of the gene has been observed and no effect of the location of the mutations on the age of onset or disease duration has been demonstrated.

\section{Role of TDP-43 in neurodegeneration}

Little is currently known about the pathogenic role of TARDBP mutations or TDP-43 pathology in neurodegeneration. Analogous to other neurodegenerative diseases, characterized by the accumulation of mis-folded protein, the critical step will be to determine whether disease results from a loss-of-function, a toxic gain-of-function or both.

The abnormal localization of TDP-43 to the cytoplasm in affected neurons in FTD and ALS, irrespective of the presence of a genetic mutation, suggests a pathogenic mechanism associated with the loss of the normal nuclear TDP-43 function in regulating transcription, splicing and mRNA stability $[29 \bullet, 57]$. In support of this hypothesis, loss of TDP-43 in human cells has been shown to induce morphological nuclear defects and increased apoptosis [58・•]. Alternatively, sequestration of TDP-43 in cellular inclusions may induce a toxic gain-of-function, independent of the basic biological role of TDP-43. A study in which TDP-43 was overexpressed in yeast suggested that only aggregating forms of TDP-43 were toxic, although the toxicity depended on an intact RNA recognition motif [59॰]. Factors that affect the normal intracellular trafficking of TDP-43, between the cytoplasm and nucleus, may predispose to both the formation of abnormal aggregates (inclusions) and the loss of nuclear localization $[60 \bullet$.

The identification of TARDBP mutations in ALS has provided important clues to the possible pathogenic mechanisms involved in TDP-43 proteinopathies. The clustering of mutations in exon 6, that encodes the highly conserved C-terminal domain, suggests that TARDBP mutations may interfere with the normal protein-protein interactions of TDP-43, affect its transport through the nuclear pore or influence its exon skipping or transcriptional repression activity $[50 \bullet, 51 \bullet \bullet$. Numerous potential phosphorylation sites have been predicted to occur in the Cterminal region of TDP-43 and some mutations, especially the six substitutions to serine and threonine residues, may increase phosphorylation and aggregation. A single mutation $\mathrm{c}$. 640A $>\mathrm{T}$ (p.D169G) was identified in TARDBP exon 4 encoding RRM1 [50•]. This mutation may abrogate the RNA binding to this region; however, because it lies outside of exon 6 and lacks evidence of segregation with disease, it may also represent a rare benign sequence variant. Finally, a study of mutant TDP-43 in cell culture and in vivo revealed increased generation of 
detergent-insoluble TDP-43 fragments that could aggregate and cause a toxic gain-of-function $[50 \bullet, 51 \bullet \cdot]$. Given the multifaceted role of TDP-43, multiple disease mechanism may well be involved.

\section{Conclusions}

The identification of pathological TDP-43 in FTLD-U and ALS confirms that these conditions are part of a disease spectrum with a common underlying biochemical mechanism. This insight has helped to clarify the relationship among the various genetic, clinical and pathological subtypes of FTD and ALS and has suggested possible mechanistic links with other neurodegenerative disorders. A better understanding of the role of TDP-43 in neurodegeneration will be crucial to the development of targeted therapeutic strategies for these conditions. Central to this process will be the use of experimental animals and cell systems, expressing pathogenic mutations in TARDBP or FTLD-U causing genes. The recent identification of elevated plasma levels of TDP-43, in some patients with neurodegenerative disease [61•], supports the possible use of TDP-43 as an in vivo biomarker to aid in diagnosis and monitoring the effects of therapy. In summary, the discovery that TDP-43 plays a central role in the pathogenesis of FTD and ALS has been a major advance towards the effective management of these devastating neurodegenerative conditions.

\section{Acknowledgments}

This work was supported by funding from the Canadian Institutes for Health Research (\#135169 and 179009), the Pacific Alzheimer Research Foundation (\#C06-01) and the National Institutes of Health (P50 AG16574).

\section{References and recommended reading}

Papers of particular interest, published within the annual period of review, have been highlighted as:

- of special interest

•• of outstanding interest

1. Mackenzie IR, Feldman HH. Ubiquitin immunohistochemistry suggests classic motor neuron disease, motor neuron disease with dementia, and frontotemporal dementia of the motor neuron disease type represent a clinicopathologic spectrum. J Neuropathol Exp Neurol 2005;64:730-739. [PubMed: 16106222]

2. Arai T, Hasegawa M, Akiyama H, et al. TDP-43 is a component of ubiquitin-positive tau-negative inclusions in frontotemporal lobar degeneration and amyotrophic lateral sclerosis. Biochem Biophys Res Commun 2006;351:602-611. [PubMed: 17084815] • One of two initial papers to identify TDP-43 as the pathological protein in FTLD-U and ALS. Demonstrates that TDP-43 co-localizes with ubiquitin-immunoreactive inclusions and that the pathological TDP-43 is composed of abnormal hyperphosphorylated C-terminal fragments.

3. Neumann M, Sampathu DM, Kwong LK, et al. Ubiquitinated TDP-43 in frontotemporal lobar degeneration and amyotrophic lateral sclerosis. Science 2006;314:130-133. [PubMed: 17023659] •• One of two initial papers to identify TDP-43 as the pathological protein in FTLD-U and ALS. Demonstrates that TDP-43 co-localizes with ubiquitin-immunoreactive inclusions and that the pathological TDP-43 is composed of abnormal hyperphosphorylated C-terminal fragments.

4. Leigh PN, Whitwell H, Garofalo O, et al. Ubiquitin-immunoreactive intraneuronal inclusions in amyotrophic lateral sclerosis. Morphology, distribution, and specificity. Brain 1991;114(Pt 2):775788. [PubMed: 1646064]

5. Lomen-Hoerth C, Anderson T, Miller B. The overlap of amyotrophic lateral sclerosis and frontotemporal dementia. Neurology 2002;59:1077-1079. [PubMed: 12370467] 
6. Okamoto K, Murakami N, Kusaka H, et al. Ubiquitin-positive intraneuronal inclusions in the extramotor cortices of presenile dementia patients with motor neuron disease. J Neurol 1992;239:426430. [PubMed: 1333007]

7. Wightman G, Anderson VE, Martin J, et al. Hippocampal and neocortical ubiquitin-immunoreactive inclusions in amyotrophic lateral sclerosis with dementia. Neurosci Lett 1992;139:269-274. [PubMed: 1376881]

8. Jackson M, Lennox G, Lowe J. Motor neurone disease-inclusion dementia. Neurodegeneration 1996;5:339-350. [PubMed: 9117546]

9. Mackenzie IR, Shi J, Shaw CL, et al. Dementia lacking distinctive histology (DLDH) revisited. Acta Neuropathol 2006;112:551-559. [PubMed: 16900341]

10. Mackenzie IR, Baborie A, Pickering-Brown S, et al. Heterogeneity of ubiquitin pathology in frontotemporal lobar degeneration: classification and relation to clinical phenotype. Acta Neuropathol 2006;112:539-549. [PubMed: 17021754]

11. Sampathu DM, Neumann M, Kwong LK, et al. Pathological heterogeneity of frontotemporal lobar degeneration with ubiquitin-positive inclusions delineated by ubiquitin immunohistochemistry and novel monoclonal antibodies. Am J Pathol 2006;169:1343-1352. [PubMed: 17003490]

12. Cairns NJ, Neumann M, Bigio EH, et al. TDP-43 in Familial and Sporadic Frontotemporal Lobar Degeneration with Ubiquitin Inclusions. Am J Pathol 2007;171:227-240. [PubMed: 17591968] •• Multi-centred international study confirming TDP-43 pathology in most sporadic FTLD-U and demonstrating that specific patterns of FTLD-U pathology correlate with each of the known genetic causes.

13. Davidson Y, Kelley T, Mackenzie IR, et al. Ubiquitinated pathological lesions in frontotemporal lobar degeneration contain the TAR DNA-binding protein, TDP-43. Acta Neuropathol 2007;113:521-533. [PubMed: 17219193] • First study to confirm the original discovery that TDP-43 pathology is characteristic of all major pathological subtypes of FTLD-U.

14. Mackenzie IR. The neuropathology and clinical phenotype of FTD with progranulin mutations. Acta Neuropathol 2007;114:49-54. [PubMed: 17458552]

15. Mackenzie IR, Bigio EH, Ince PG, et al. Pathological TDP-43 distinguishes sporadic amyotrophic lateral sclerosis from amyotrophic lateral sclerosis with SOD1 mutations. Ann Neurol 2007;61:427434. [PubMed: 17469116] • Large multicentred series demonstrating that pathological TDP-43 is characteristic of all sporadic ALS and familial ALS without SOD1 mutations, but is absent in cases with SOD1 mutations. Suggests that SOD1 mutations may cause neurodegeneration by a different mechanism.

16. Tan CF, Eguchi H, Tagawa A, et al. TDP-43 immunoreactivity in neuronal inclusions in familial amyotrophic lateral sclerosis with or without SOD1 gene mutation. Acta Neuropathol 2007;113:535542. [PubMed: 17333220]

17. Neumann M, Mackenzie IR, Cairns NJ, et al. TDP-43 in the ubiquitin pathology of frontotemporal dementia with VCP gene mutations. J Neuropathol Exp Neurol 2007;66:152-157. [PubMed: 17279000]

18. Neumann M, Kwong LK, Truax AC, et al. TDP-43-positive white matter pathology in frontotemporal lobar degeneration with ubiquitin-positive inclusions. J Neuropathol Exp Neurol 2007;66:177-183. [PubMed: 17356379] • Provides first detailed description of TDP-43 inclusions in glial cells in FTLDU.

19. Geser F, Brandmeir NJ, Kwong LK, et al. Evidence of multisystem disorder in whole-brain map of pathological TDP-43 in amyotrophic lateral sclerosis. Arch Neurol 2008;65:636-641. [PubMed: 18474740]

20. Hatanpaa KJ, Bigio EH, Cairns NJ, et al. TAR DNA-binding protein 43 immunohistochemistry reveals extensive neuritic pathology in FTLD-U: a midwest-southwest consortium for FTLD study. J Neuropathol Exp Neurol 2008;67:271-279. [PubMed: 18379440]

21. Hasegawa M, Arai T, Nonaka T, et al. Phosphorylated TDP-43 in frontotemporal lobar degeneration and amyotrophic lateral sclerosis. Ann Neurol. 2008 - Antibodies directed against phosphorylated epitopes of pathological TDP-43 were developed and shown to be more sensitive and specific for the pathological inclusions. All the pathological changes in FTLD-U and ALS were shown to contain TDP-43 that is abnormally phosphorylated. 
22. Igaz LM, Kwong LK, Xu Y, et al. Enrichment of C-terminal fragments in TAR DNA-binding protein-43 cytoplasmic inclusions in brain but not in spinal cord of frontotemporal lobar degeneration and amyotrophic lateral sclerosis. Am J Pathol 2008;173:182-194. [PubMed: 18535185] • Antibodies specific for the $\mathrm{C}$-terminal and $\mathrm{N}$-terminal region were used to demonstrate that the neuronal inclusions in different anatomical regions may contain different TDP-43 fragments. This suggests that the process of inclusion formation may vary in different neuronal populations.

23. Buratti E, Dork T, Zuccato E, et al. Nuclear factor TDP-43 and SR proteins promote in vitro and in vivo CFTR exon 9 skipping. Embo J 2001;20:1774-1784. [PubMed: 11285240]

24. Wang HY, Wang IF, Bose J, et al. Structural diversity and functional implications of the eukaryotic TDP gene family. Genomics 2004;83:130-139. [PubMed: 14667816]

25. Ou SH, Wu F, Harrich D, et al. Cloning and characterization of a novel cellular protein, TDP-43, that binds to human immunodeficiency virus type 1 TAR DNA sequence motifs. J Virol 1995;69:35843596. [PubMed: 7745706]

26. Mercado PA, Ayala YM, Romano M, et al. Depletion of TDP 43 overrides the need for exonic and intronic splicing enhancers in the human apoA-II gene. Nucleic Acids Res 2005;33:6000-6010. [PubMed: 16254078]

27. Buratti E, Brindisi A, Giombi M, et al. TDP-43 binds heterogeneous nuclear ribonucleoprotein A/B through its C-terminal tail: an important region for the inhibition of cystic fibrosis transmembrane conductance regulator exon 9 splicing. J Biol Chem 2005;280:37572-37584. [PubMed: 16157593]

28. Wang IF, Reddy NM, Shen CK. Higher order arrangement of the eukaryotic nuclear bodies. Proc Natl Acad Sci U S A 2002;99:13583-13588. [PubMed: 12361981]

29. Buratti E, Baralle FE. Multiple roles of TDP-43 in gene expression, splicing regulation, and human disease. Front Biosci 2008;13:867-878. [PubMed: 17981595] • Detailed review of normal function of TDP-43 and relationship to human disease.

30. Wang IF, Wu LS, Chang HY, et al. TDP-43, the signature protein of FTLD-U, is a neuronal activityresponsive factor. J Neurochem 2008;105:797-806. [PubMed: 18088371]

31. Mackenzie IR, Foti D, Woulfe J, et al. Atypical frontotemporal lobar degeneration with ubiquitinpositive, TDP-43-negative neuronal inclusions. Brain 2008;131:1282-1293. [PubMed: 18362096] • First detailed description of a new clinicopathological subtype of FTLD characterized by severe, early-onset psychobehavioural changes and FTLD-U pathology that is negative for TDP-43. Indicates that in a significant minority of FTLD-U, the ubiquitinated pathological protein is something other than TDP-43.

32. Roeber S, Mackenzie IR, Kretzschmar HA, et al. TDP-43-negative FTLD-U is a significant new clinico-pathological subtype of FTLD. Acta Neuropathol. 2008

33. Holm IE, Englund E, Mackenzie IR, et al. A reassessment of the neuropathology of frontotemporal dementia linked to chromosome 3. J Neuropathol Exp Neurol 2007;66:884-891. [PubMed:

17917582] - Review of the neuropathology in the Danish family with FTD linked to chromosome 3 caused by mutation in the CHMP2B gene. Identifies unique subtype of FTLD-U with ubiquitinimmunoreactive inclusions in dentate granule cells that are negative for TDP-43.

34. Robertson J, Sanelli T, Xiao S, et al. Lack of TDP-43 abnormalities in mutant SOD1 transgenic mice shows disparity with ALS. Neurosci Lett 2007;420:128-132. [PubMed: 17543992] • Confirms absence of TDP-43 pathology in SOD1 mouse model for ALS. Contradicts other studies by suggesting that some cases of human ALS with SOD1 mutations have at least mild TDP-43 pathology.

35. Geser F, Winton MJ, Kwong LK, et al. Pathological TDP-43 in parkinsonism-dementia complex and amyotrophic lateral sclerosis of Guam. Acta Neuropathol 2008;115:133-145. [PubMed: 17713769] - One of two studies showing that extensive TDP-43 pathology is a characteristic feature of PDC/ ALS of Guam.

36. Hasegawa M, Arai T, Akiyama H, et al. TDP-43 is deposited in the Guam parkinsonism-dementia complex brains. Brain 2007;130:1386-1394. [PubMed: 17439983] • One of two studies showing that extensive TDP-43 pathology is a characteristic feature of PDC/ALS of Guam.

37. Amador-Ortiz C, Lin WL, Ahmed Z, et al. TDP-43 immunoreactivity in hippocampal sclerosis and Alzheimer's disease. Ann Neurol 2007;61:435-445. [PubMed: 17469117] •• First study to demonstrate TDP-43 pathology in a significant proportion of cases with Alzheimer's disease. 
38. Probst A, Taylor KI, Tolnay M. Hippocampal sclerosis dementia: a reappraisal. Acta Neuropathol 2007;114:335-345. [PubMed: 17639426]

39. Freeman SH, Spires-Jones T, Hyman BT, et al. TAR-DNA binding protein 43 in Pick disease. J Neuropathol Exp Neurol 2008;67:62-67. [PubMed: 18091558]

40. Uryu K, Nakashima-Yasuda H, Forman MS, et al. Concomitant TAR-DNA-binding protein 43 pathology is present in Alzheimer disease and corticobasal degeneration but not in other tauopathies. J Neuropathol Exp Neurol 2008;67:555-564. [PubMed: 18520774]

41. Higashi S, Iseki E, Yamamoto R, et al. Concurrence of TDP-43, tau and alpha-synuclein pathology in brains of Alzheimer's disease and dementia with Lewy bodies. Brain Res 2007;1184C:284-294. [PubMed: 17963732]

42. Nakashima-Yasuda H, Uryu K, Robinson J, et al. Co-morbidity of TDP-43 proteinopathy in Lewy body related diseases. Acta Neuropathol (Berl) 2007;114:221-229. [PubMed: 17653732]

43. Goate A, Chartier-Harlin MC, Mullan M, et al. Segregation of a missense mutation in the amyloid precursor protein gene with familial Alzheimer's disease. Nature 1991;349:704-706. [PubMed: 1671712]

44. Hutton M, Lendon CL, Rizzu P, et al. Association of missense and 5'-splice-site mutations in tau with the inherited dementia FTDP-17. Nature 1998;393:702-705. [PubMed: 9641683]

45. Polymeropoulos MH, Lavedan C, Leroy E, et al. Mutation in the alpha-synuclein gene identified in families with Parkinson's disease. Science 1997;276:2045-2047. [PubMed: 9197268]

46. Gijselinck I, Sleegers K, Engelborghs S, et al. Neuronal inclusion protein TDP-43 has no primary genetic role in FTD and ALS. Neurobiol Aging. 2007

47. Rollinson S, Snowden JS, Neary D, et al. TDP-43 gene analysis in frontotemporal lobar degeneration. Neurosci Lett. 2007

48. Schumacher A, Friedrich P, Diehl-Schmid J, et al. No association of TDP-43 with sporadic frontotemporal dementia. Neurobiol Aging. 2007

49. Gitcho MA, Baloh RH, Chakraverty S, et al. TDP-43 A315T mutation in familial motor neuron disease. Ann Neurol. 2008 - First report of a missense mutation in TARDBP segregating with disease in an ALS family. Provides evidence for a direct role of TDP-43 in neurodegeneration.

50. Kabashi E, Valdmanis PN, Dion P, et al. TARDBP mutations in individuals with sporadic and familial amyotrophic lateral sclerosis. Nat Genet. 2008 Extensive TARDBP mutation screening reporting 8 different mutations in familial and sporadic ALS patients with clustering in TDP-43 C-terminal region.

51. Sreedharan J, Blair IP, Tripathi VB, et al. TDP-43 mutations in familial and sporadic amyotrophic lateral sclerosis. Science 2008;319:1668-1672. [PubMed: 18309045] •• First study reporting missense mutations in TARDBP in familial and sporadic ALS patients. Provides in vivo evidence that mutant forms of TDP-43 are toxic.

52. Yokoseki A, Shiga A, Tan CF, et al. TDP-43 mutation in familial amyotrophic lateral sclerosis. Ann Neurol 2008;63:538-542. [PubMed: 18438952]

53. Van Deerlin VM, Leverenz JB, Bekris LM, et al. TARDBP mutations in amyotrophic lateral sclerosis with TDP-43 neuropathology: a genetic and histopathological analysis. Lancet Neurol 2008;7:409416. [PubMed: 18396105] - Provides first detailed description of TDP-43 neuropathology in affected family members carrying TARDBP mutations.

54. Guerreiro RJ, Schymick JC, Crews C, et al. TDP-43 is not a common cause of sporadic amyotrophic lateral sclerosis. PLoS ONE 2008;3:e2450. [PubMed: 18545701]

55. Traynor BJ, Codd MB, Corr B, et al. Clinical features of amyotrophic lateral sclerosis according to the El Escorial and Airlie House diagnostic criteria: A population-based study. Arch Neurol 2000;57:1171-1176. [PubMed: 10927797]

56. Zoccolella S, Beghi E, Palagano G, et al. Signs and symptoms at diagnosis of amyotrophic lateral sclerosis: a population-based study in southern Italy. Eur J Neurol 2006;13:789-792. [PubMed: 16834713]

57. Strong MJ, Volkening K, Hammond R, et al. TDP43 is a human low molecular weight neurofilament (hNFL) mRNA-binding protein. Mol Cell Neurosci 2007;35:320-327. [PubMed: 17481916]

58. Ayala YM, Misteli T, Baralle FE. TDP-43 regulates retinoblastoma protein phosphorylation through the repression of cyclin-dependent kinase 6 expression. Proc Natl Acad Sci U S A 2008;105:3785- 
3789. [PubMed: 18305152] • Excellent cell biological paper focused on the identification of TDP-43 regulated transcripts. Indicates that normal TDP-43 function is critical for cell survival.

59. Johnson BS, McCaffery JM, Lindquist S, et al. A yeast TDP-43 proteinopathy model: Exploring the molecular determinants of TDP-43 aggregation and cellular toxicity. Proc Natl Acad Sci U S A 2008;105:6439-6444. [PubMed: 18434538] - Description of a yeast TDP-43 proteinopathy model recapitulating many features of human TDP-43 proteinopathies. Provides opportunity to study TDP-43 aggregation and its cellular consequences.

60. Winton MJ, Igaz LM, Wong MM, et al. Disturbance of nuclear and cytoplasmic Tar DNA binding protein (TDP-43) induces disease-like redistribution, sequestration and aggregate formation. J Biol Chem. 2008 - Interesting cell culture study suggesting that perturbation of nuclear and cytoplasmic trafficking of TDP-43 leads to the formation of cytoplasmic TDP-43 aggregates similar to those observed in FTD and ALS patients.

61. Foulds P, McAuley E, Gibbons L, et al. TDP-43 protein in plasma may index TDP-43 brain pathology in Alzheimer's disease and frontotemporal lobar degeneration. Acta Neuropathol. 2008 • First demonstration that TDP-43 levels can be measured in plasma and are elevated in some patients with neurodegenerative disease.

62. Mackenzie IR, Rademakers R. The molecular genetics and neuropathology of frontotemporal lobar degeneration: recent developments. Neurogenetics 2007;8:237-248. [PubMed: 17805587] 


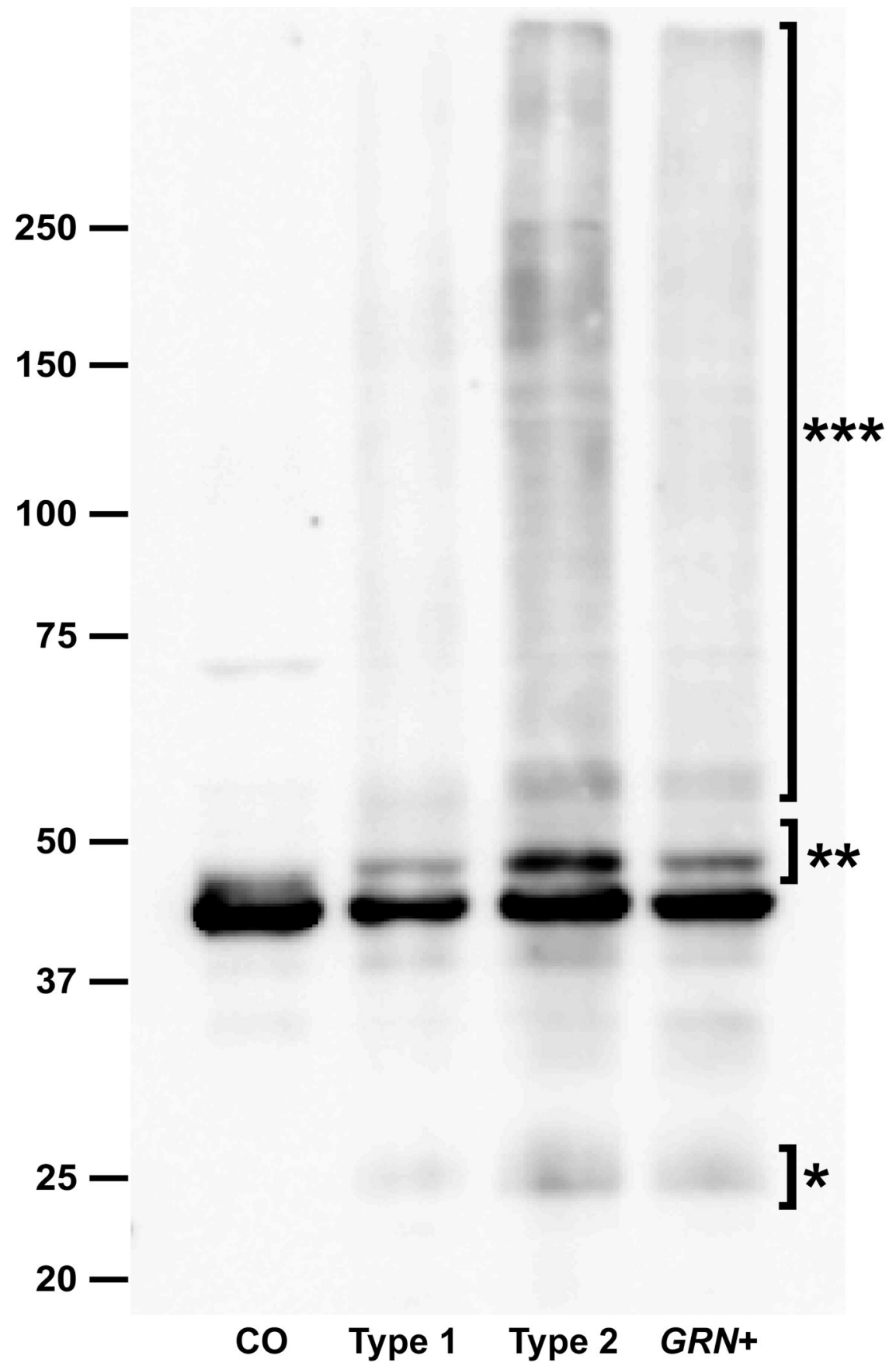

Fig. 1. TDP-43 immunohistochemistry in frontotemporal lobar degeneration with ubiquitinated inclusions (FTLD-U) and amyotrophic lateral sclerosis (ALS)

A polyclonal antibody against TDP-43 labels neuronal inclusions in (a) neocortex and (b) hippocampus in FTLD-U and (c) lower motor neurons in ALS. Note that cells with inclusions show loss of normal diffuse nuclear staining. TDP-43 immunohistochemistry. Scale bar; (a,b) $20 \mu \mathrm{m}$, (c) $15 \mu \mathrm{m}$. 

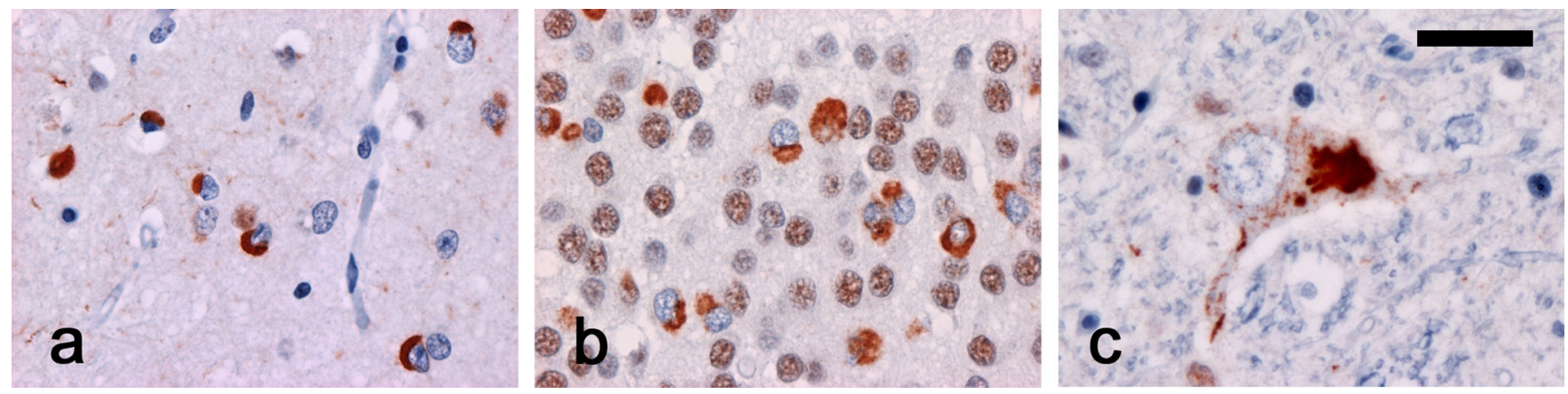

Fig. 2. Biochemical analysis of pathologic TDP-43 in frontotemporal lobar degeneration with ubiquitinated inclusions (FTLD-U)

Immunoblot analysis of urea fraction from post mortem FTLD-U brain tissue using antiTDP-43 antibody demonstrates pathological bands at $\sim 25 \mathrm{kD}(*), \sim 45 \mathrm{kD}(* *)$ and a high $\mathrm{M}_{\mathrm{r}}$ smear (***). CO, control; type 1, sporadic FTLD-U with type 1 pathology; type 2, sporadic FTLD-U with type 2 pathology; GRN+, familial FTLD-U with progranulin mutation and type 3 pathology [11]. A similar immunoblot pattern is seen in studies of post mortem spinal cord tissue from cases of amyotrophic lateral sclerosis. Previously published and reprinted with permission [62]. 

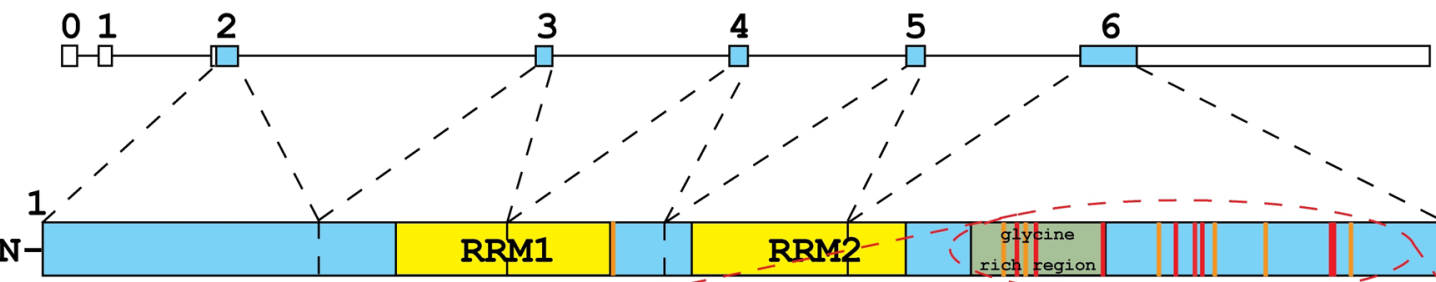

RRM2
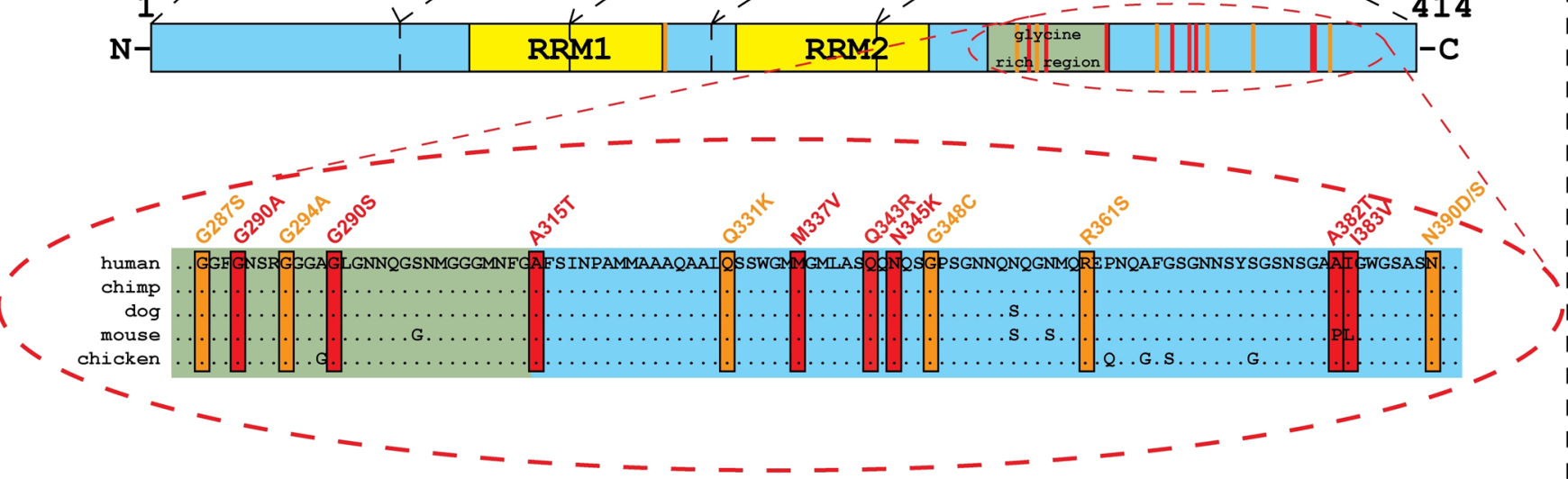

Fig. 3. Missense mutations in the gene encoding TDP-43 (TARDBP) cause ALS

Schematic overview of the TDP-43 gene (TARDBP) and protein structure with locations of conserved domains (protein numbering according to the largest isoforms NP_031401.1) (top). Protein sequence alignment shows strong conservation in the C-terminal region of TDP-43 (bottom). Colored boxes indicate the position of TARDBP mutations identified in sporadic (orange) and familial (red) ALS patients. RRM= RNA recognition motif. 